\title{
Aceptación del Modelo de EA "Vaula" por parte del alumnado
}

Rosales, Consuelo ${ }^{a}$, Molina, Francisca ${ }^{b}$ y Huertas, Ana $^{c}$

aUniversidad de Jaén, mrosales@ujaen.es, bUniversidad de Jaén, mfmolina@ujaen.es y ${ }^{c}$ Universidad de Jaén, mhuertas@ujaen.es.

\section{\$EWWFW}

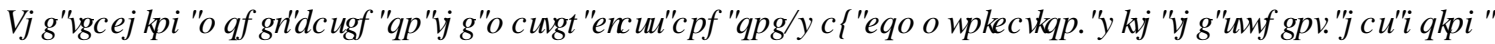

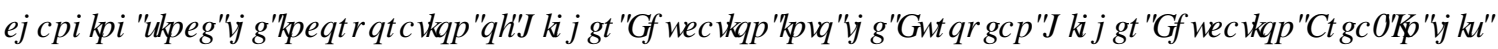

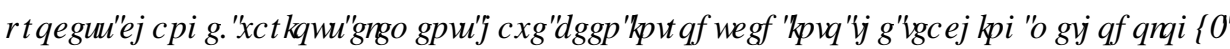

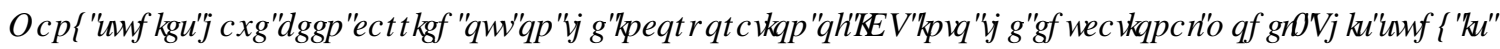

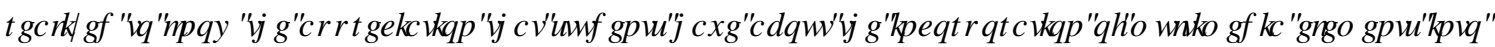

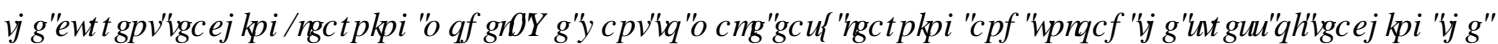

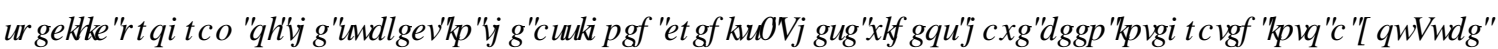

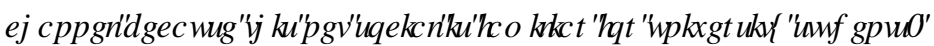

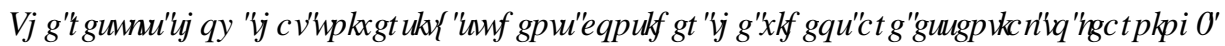

[

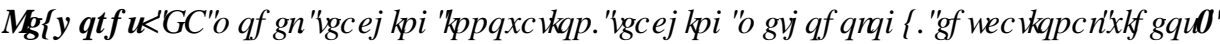

\section{HXP HQ}

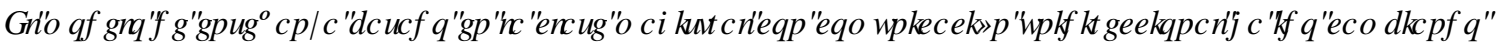

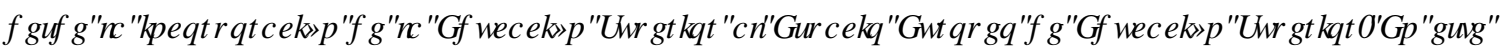

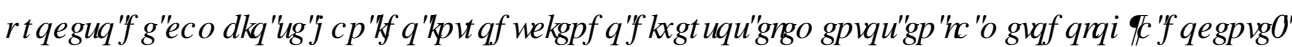

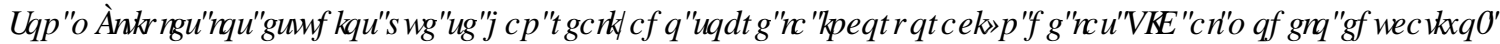

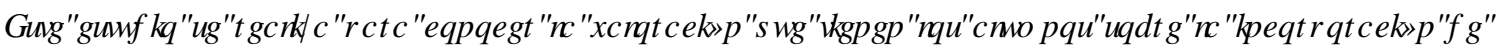

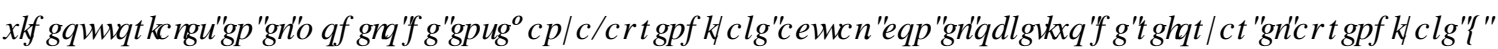

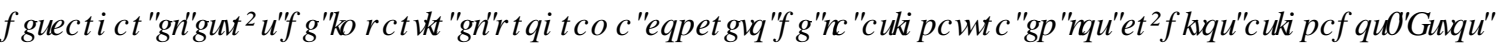

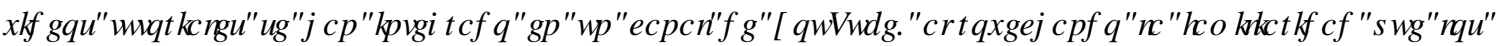

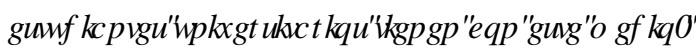

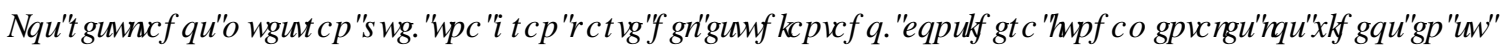
DSLHQG]DNAIL

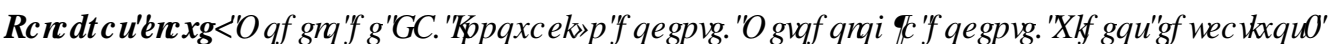




\section{Introducción}

El interés creciente del alumnado en la búsqueda y utilización de videos, con el fin de lograr el aprendizaje de los conceptos y procedimientos matemáticos, nos ha llevado, como docentes del Departamento de Matemáticas de la Universidad de Jaén, a diseñar un modelo mixto de enseñanza aprendizaje, al que hemos denominado "Vaula", para la docencia de los contenidos de Cálculo Numérico que se imparten en las asignaturas que están adscritas a nuestro Departamento y que mostramos en la tabla 1

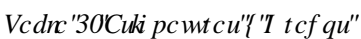

\begin{tabular}{cc}
\hline Asignatura & Grado \\
\hline Análisis y Métodos Numéricos & Grado en Ingeniería Informática \\
Matemáticas II & Grado en Ingeniería de Organización Industrial \\
Matemáticas II & Grado en Ingeniería Mecánica \\
Matemáticas II & Grado en Ingeniería Eléctrica \\
Matemáticas II & Doble grado en Ingeniería Eléctrica e Ingeniería Mecánica \\
Matemáticas II & Doble grado en Ingeniería Eléctrica e Ingeniería Electrónica \\
& Industrial \\
Matemáticas II & Doble grado en Ingeniería Mecánica e Ingeniería de Organización \\
Matemáticas II & Doble grado en Ingeniería Mecánica e Ingeniería Electrónica \\
& Industrial \\
\hline
\end{tabular}

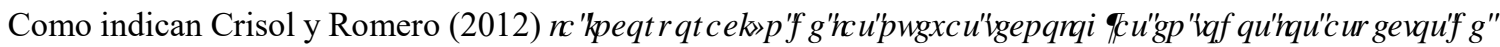

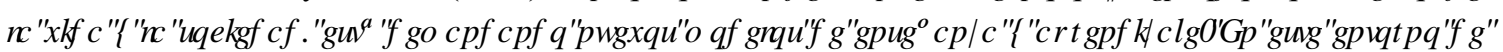

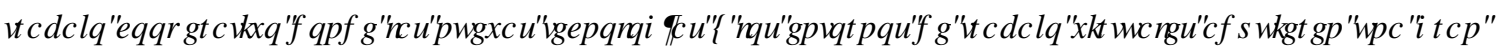

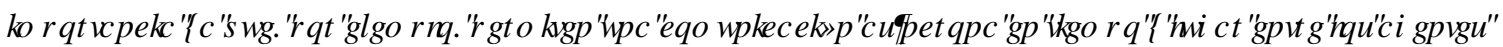

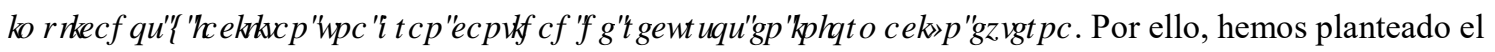
modelo de EA "Vaula" de forma que se complementen las actividades docentes clásicas, como clases magistrales impartidas de forma síncrona y apoyo en la bibliografía en formato escrito, con nuevas herramientas didácticas como son los videos, páginas web y guías diseñadas para facilitar el aprendizaje y autoaprendizaje del alumno.

Después de analizar las peculiaridades de los contenidos de Cálculo Numérico que debemos enseñar y transmitir a nuestros alumnos, los objetivos de aprendizaje que pretendemos que alcancen, las dificultades detectadas en la EA de estos contenidos y la tipología y aplicabilidad de los procedimientos de esta materia hemos diseñado un modelo didáctico estructurado en torno a los siguientes elementos:

- Clases magistrales síncronas en las que se explican los fundamentos teóricos en los que se basan los métodos y procedimientos numéricos de los temas a abordar.

- Clases de prácticas con ordenador, síncronas, en las que se trabaja la implantación de los métodos numéricos con software adecuado. En nuestra Universidad utilizamos Wolfram Mathematica. 
- Un canal de YouTube, al que hemos denominado "Cálculo Numérico para Ingenieros" y en el que se ofrece un conjunto estructurado de vídeos cortos, que abordan y resuelven distintos tipos de ejercicios de aplicación y problemas. Están organizados según temática, grado de complejidad y aplicabilidad a la ingeniería y la ciencia.

- Guía de orientación del recorrido educativo y secuenciación que el alumno puede seguir en su trabajo autónomo de aprendizaje.

- Una página web, "Algorítmica Numérica", como una herramienta de enseñanza que permite integrar la información, tanto la necesaria como la complementaria, para el alumnado en su proceso de aprendizaje.

- Tutorías síncronas online que apoyan al alumnado en la resolución de dudas y consolidación de su aprendizaje.

Para determinar la demanda, validez y confiabilidad, y medir el grado de aceptación del modelo que presentamos, junto con los elementos que lo componen, hemos interactuado con 430 alumnos que están matriculados en nuestras asignaturas. La información la hemos recogido por varias vías:

- Planteando cuestionarios al alumnado para indagar sobre las necesidades educativas, preferencias de modelo, utilidad y aceptación de nuestras distintas propuestas.

- Visionado y puntuación de los videos, recogidos mediante formularios.

- Interacción con el canal de YouTube.

- Detección, en tutorías o por interpelaciones del alumnado, de los problemas o carencias que se encuentran en el aprendizaje de los contenidos.

- Retroalimentación o crítica constructiva del alumnado.

En la actualidad contamos con 35 videos repartidos del siguiente modo entre las unidades didácticas que componen la totalidad de la asignatura:

1.- Resolución Numérica de ecuaciones no lineales. (9)

2.- Aproximación de funciones. (9)

3.- Integración Numéricas. (9)

4.- Resolución Numérica de PVI. (8)

\section{Objetivos}

El objetivo fundamental de esta investigación es conocer el grado de aceptación que tiene el modelo de EA "Vaula" en nuestro alumnado, pero también consideramos de gran valor plantear los siguientes objetivos complementarios que nos permitan fundamentar el avance en la implantación de este nuevo modelo:

- Fundamentar la innovación docente que llevamos a cabo mediante el modelo de EA "Vaula" interactuando directamente con nuestro alumnado para conocer si nuestra nueva oferta didáctica da una mejor respuesta a sus necesidades en el aprendizaje.

- Profundizar en el conocimiento de los procedimientos que el alumnado emplea en el trabajo autónomo que realiza para aprender los contenidos y para entrenar las destrezas matemáticas que le permiten resolver problemas.

- Descubrir las expectativas que tiene el alumnado cuando se le presentan nuevas herramientas complementarias para el aprendizaje. 
- Evaluar los elementos del modelo y su cohesión, detectando utilidades, opiniones, valoraciones y aportaciones del alumnado con la finalidad de reorientar, ajustar y ampliar nuestro trabajo.

Para lograr estos objetivos definimos las siguientes acciones:

- Diseño del formulario a fin de conocer las expectativas que generaría la implantación de este nuevo modelo, entre nuestro alumnado.

En este cuestionario no planteamos preguntas directas sobre el modelo de EA, sino que realizamos preguntas concretas sobre actividades que el alumno debería trabajar, para, posteriormente, en el análisis de datos, poder hacer la interpretación adecuada de los mismos.

El motivo de esta decisión fue con el objeto de recoger el mayor número de respuestas posibles, ya que por experiencia sabemos que, si los alumnos no comprenden las preguntas o las ven alejadas de su realidad, no responden.

- Recogida de datos de este cuestionario, mediante envío de formulario Google form.

- Creación del canal de YouTube y subida del material con el que los alumnos deben interactuar en este modelo.

- Diseño del cuestionario para recoger información de la utilidad de la implantación de este modelo. $\mathrm{Al}$ igual que el formulario anterior, se diseñó con preguntas concretas y específicas relacionadas con la inclusión de las herramientas, con las que cuenta este modelo, en el estudio individual que realiza el alumnado.

- Recogida de datos de este cuestionario, mediante envío de formulario Google form.

\section{Desarrollo de la Innovación}

Como hemos comentado en el apartado anterior, el principal objetivo de esta investigación es analizar la aceptación del nuevo modelo de EA, para lo que pasamos a la ejecución de las acciones relatadas anteriormente.

Este trabajo de valoración y análisis de la nueva metodología, se inició con una etapa previa en la que recabamos información sobre la necesidad, utilidad e impacto que tendrían nuestras aportaciones, de nuevas metodologías docentes, en el aprendizaje de nuestro alumnado, continuamos con la etapa de implementación en la que consolidamos la puesta en marcha del canal y activamos la página web. Finalizamos el estudio con la etapa de valoración de resultados y retroalimentación.

\subsection{Etapa previa}

En esta etapa, nos planteamos como objetivo, conocer el impacto que tendría contar con un canal de YouTube y una guía de recorrido educativo, en nuestra metodología docente ¿Qué aceptación tendría entre el alumnado?. Para ello realizamos una encuesta inicial al alumnado de las asignaturas 0 DUP $i$ UFDV, , de

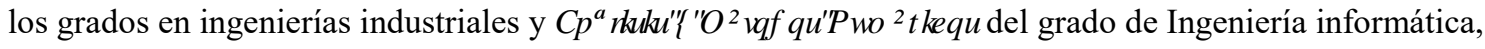
430 alumnos en total.

Con este formulario queríamos obtener la valoración personal, de cada estudiante, en relación a la incidencia que tendrían la incorporación de las nuevas herramientas, incluidas en este nuevo modelo, en el aprendizaje de contenidos y procedimientos matemáticos, así como la utilidad de la propia metodología de EA. 
Este formulario fue breve y conciso. Se plantearon 5 cuestiones y se facilitó espacio abierto para aportaciones, libres, del alumnado. La brevedad se justificó por favorecer la cumplimentación y aumentar, así, el porcentaje de respuesta y la concisión para enfocar nuestra propuesta con claridad.

Las cuestiones esenciales incidieron sobre la utilidad de:

- Disponer de vídeos con la resolución de los problemas que se planteaban de antemano en las relaciones de ejercicios.

- Visionar videos "a priori”, con contenidos introductorios antes de la sesión de clase, para orientar y enfocar el trabajo en dicha sesión.

- Visionar videos "a posteriori”, después de las explicaciones de clase para reforzar lo expuesto.

- Utilidad de un modelo mixto en el que se complementan trabajo autónomo-videos y clases

- Aportar, además, los problemas resueltos en papel.

Resultó de gran valor, poder contar con las impresiones del alumnado para detectar, en el aspecto metodológico, la preferencia del modelo de interrelación entre las actividades síncronas utilizadas en la metodología tradicional y las actividades complementarias y de autoaprendizaje, que incluimos en nuestra propuesta. En este aspecto, a la luz de las respuestas relacionadas con la utilidad del modelo mixto de EA, se buscaría encontrar un equilibrio entre la orientación para llevar a cabo el autoaprendizaje y el permitir que desarrollasen su propia autonomía, independencia y gestión de su propio aprendizaje.

\subsection{Etapa de materialización y construcción del Canal}

Tras los planteamientos iniciales y la selección de la tipología de contenido, se generaron videos que se subieron al canal. El contenido se estructuró con el criterio de nivel de dificultad y aplicabilidad de los procedimientos desarrollados: básico (centrado en los fundamentos matemáticos de los métodos), intermedio (aplicación de los métodos a la resolución de problemas en su área de desarrollo profesional, sin demasiada complejidad), avanzado o de generalización (se aplican los procedimientos aprendidos a nuevas situaciones en el ámbito de la ingeniería).

Se difundió la información relativa a las nuevas herramientas, incluidas en el modelo, por medio de comunicaciones a los grupos de alumnos de los que somos docentes y los interpelamos para que visualizaran los videos e interactuaran con el canal. También les facilitamos la relación de contenidos (teoría y problemas) que podían encontrar en el canal.

En esta etapa pretendíamos que el estudiantado, interactuara con la nueva herramienta que estábamos implementado en nuestro modelo, para posteriormente conocer la utilidad y la incidencia de la misma en el aprendizaje de nuestro alumnado.

\subsection{Etapa de retroalimentación y valoración del modelo}

Una vez constatada la aceptación del modelo era necesario medir la eficacia y la calidad de las herramientas virtuales que se estaban proporcionando al alumnado. Con esta finalidad se diseñó una segunda encuesta, propuesta recientemente, mediante un formulario Google.

- Se plantearon cuestiones para que el alumnado valorara, por un lado, para cada video, su aspecto formal: el formato que se le había dado, la duración, la calidad de audio y de imagen. Por otro lado, se buscó la valoración del contenido, el discurso y la adecuación de contenidos y de nivel.

- Se preguntó sobre la utilidad de los videos como herramienta de ayuda en su proceso de aprendizaje y también la valoración del canal, con una escala numérica de 0 a 10.

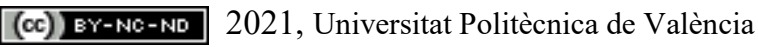

CRQJUHRL, Q5 HGD००स० 
- Se ofreció un espacio abierto para comentarios.

Se analizaron las respuestas en varios sentidos:

- Buscando contrastar las valoraciones de la experiencia real, con las expectativas (medidas en la primera encuesta) que había generado la puesta en marcha de este proyecto.

- Para conocer la aceptación, de un modo cuantitativo, por parte del alumnado del espacio y la estructura en el que se ofrecen los contenidos en los videos.

- Recopilando y sintetizando las aportaciones libres del alumnado.

\section{Resultados}

Se muestran los resultados detallados que hemos recogido y analizado en el contexto del desarrollo de la investigación que se ha relatado. Desde los inicialmente obtenidos, en una etapa previa para detectar las preferencias y necesidades de nuevas herramientas útiles en el autoaprendizaje del alumnado, siguiendo con los que se observan tras la puesta en marcha del canal de YouTube, hasta los que recogemos en un reciente formulario con la finalidad de valorar el modelo, constatar su aceptación y poner en marcha el proceso de revisión necesario.

\subsection{Encuesta sobre la valoración del modelo}

Esta encuesta se hizo con el fin de analizar las expectativas que generaría la inclusión de videos educativos en la metodología docente que estábamos utilizando para la EA de los contenidos de Cálculo Numérico. La encuesta se envió a 430 alumnos, con el perfil ya relatado en apartado anteriores, y respondieron el 60,23\% de los encuestados, es decir, 259 alumnos.

El análisis de las respuestas a esta encuesta es el siguiente:

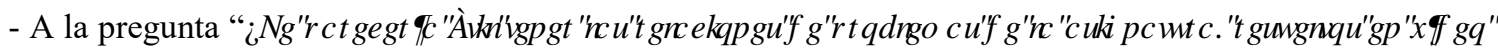

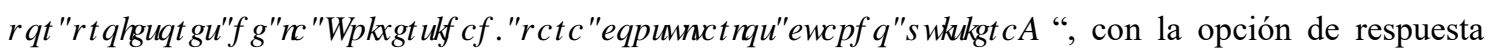
SI/NO/No sabe no contesta, un $96,7 \%$ del alumnado se mostró a favor y ningún alumno se abstuvo en la respuesta (Fig.1).

प

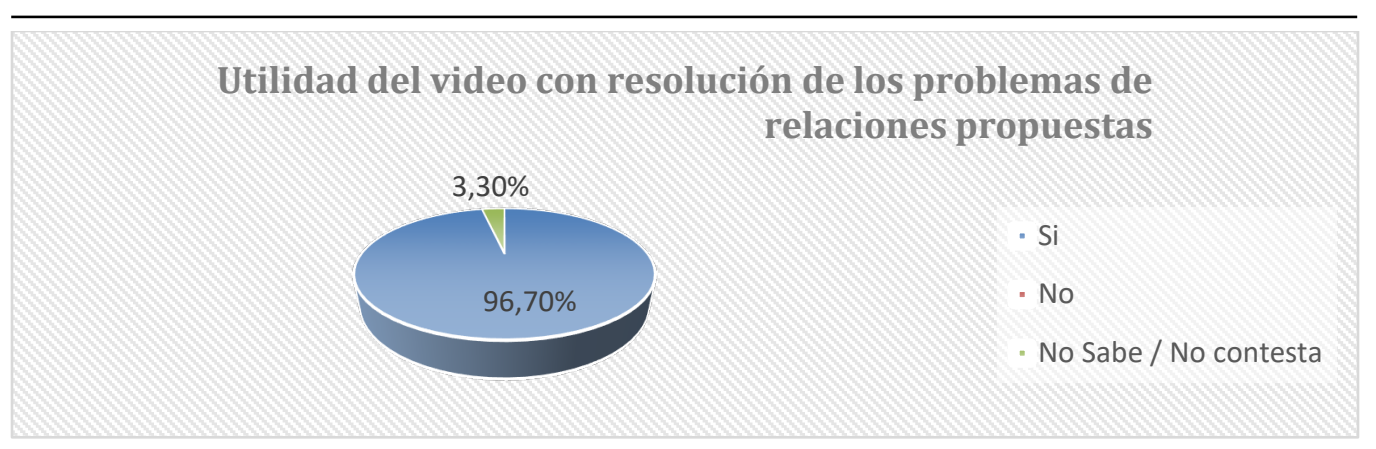

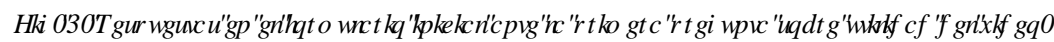

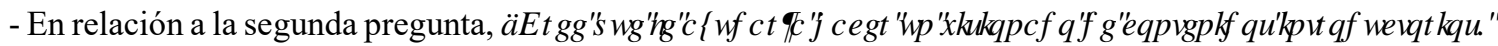

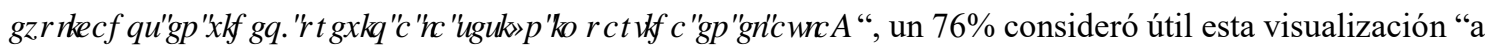
priori”, el 15,20\% considera que no sería de utilidad y el 8,7\% no sabe o no contesta la pregunta. (Fig. 2) 


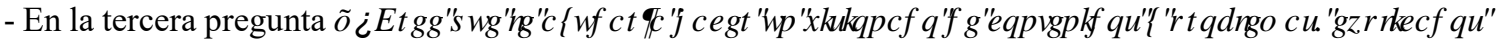

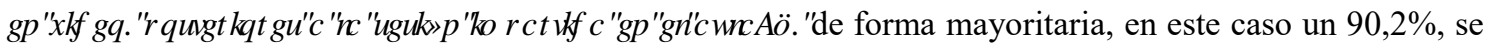
mostró a favor de esta visualización "a posteriori” y un 5,4\% no sabe o no contesta. (Fig. 2).

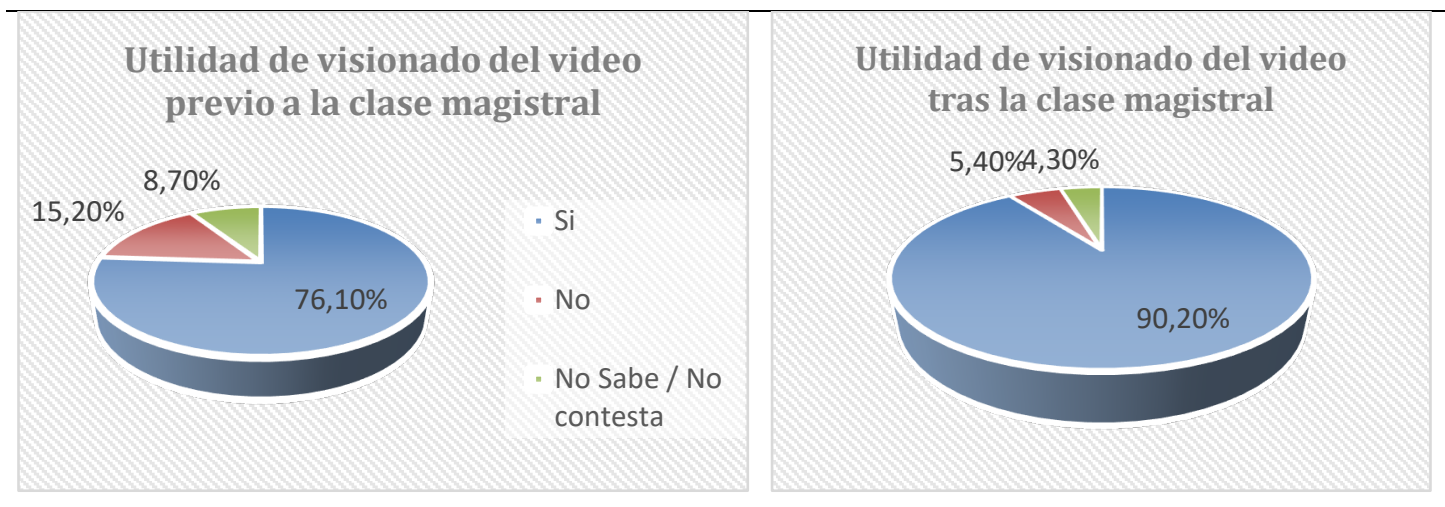

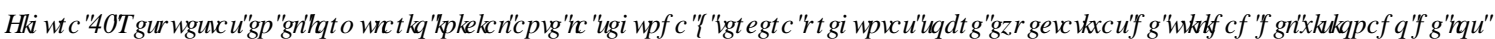

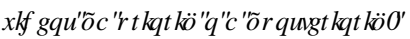

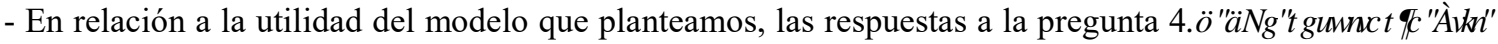

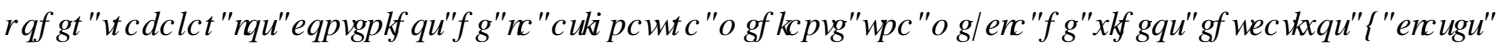
SUMHQFDON $N^{\prime}$, un $82 \%$ ve utilidad en el uso de esta herramienta y un $7,6 \%$ cree que no sería útil.

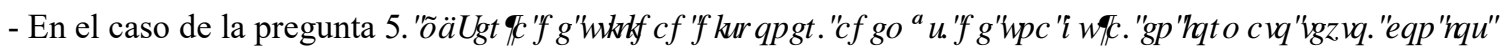

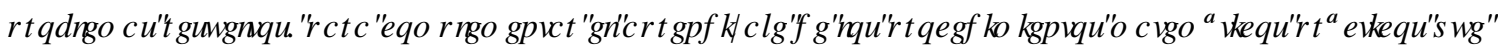

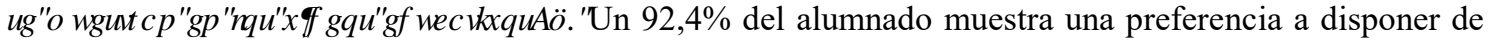
material de ayuda y orientación (Fig. 3).

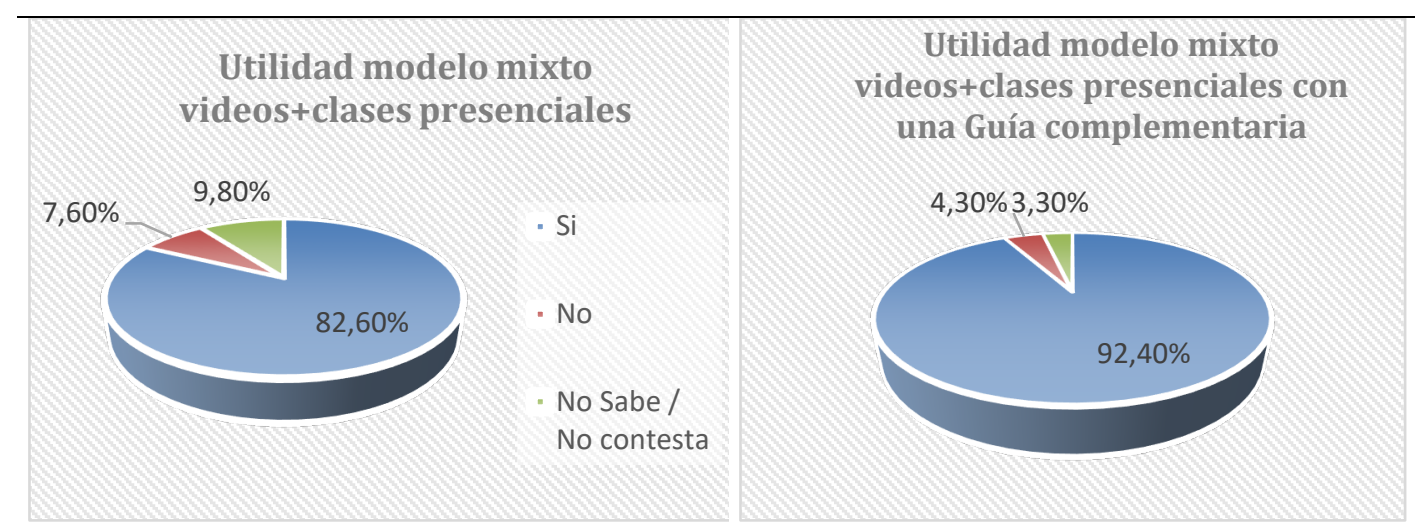

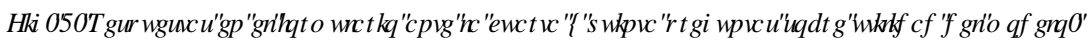

\subsection{Encuesta de valoración del canal}

El segundo formulario, que cumplimentó el estudiantado, fue enfocado a medir la eficacia y la calidad de las herramientas que se habían puesto a su disposición, con la finalidad de orientar un necesario proceso de 
reajuste del modelo tras la etapa inicial de su puesta en marcha. Este cuestionario fue enviado a 430 alumnos y respondieron 310 , es decir, un $72,09 \%$.

- Las respuestas que valoraban la claridad explicativa (de deficiente a excelente), como respuesta a la

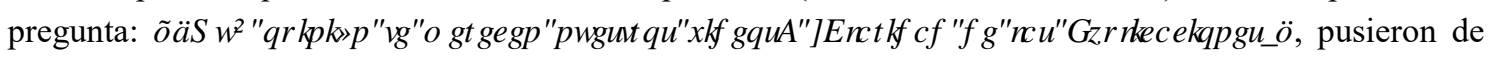
manifiesto una aceptación más que notable del modelo en relación a la forma de explicar los contenidos (Fig. 5).

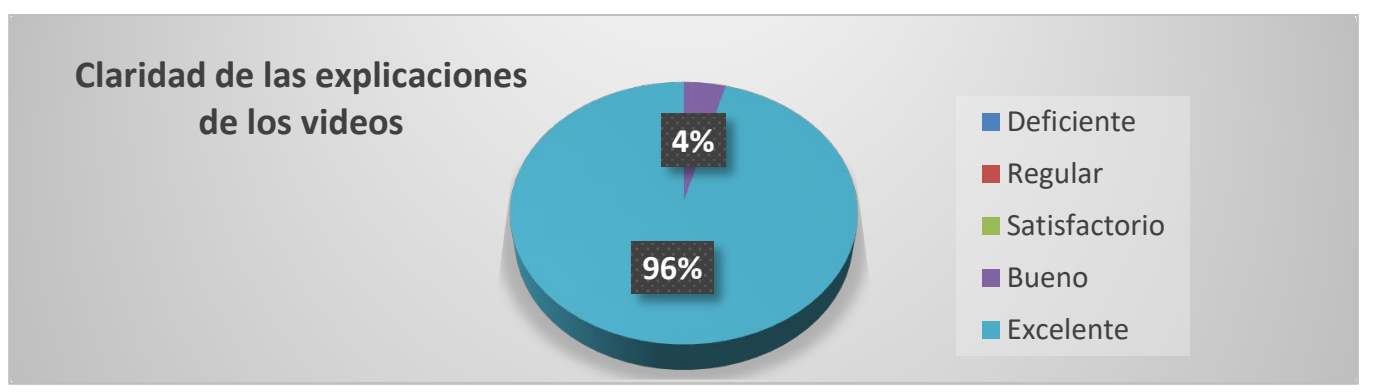

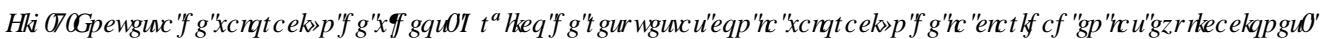

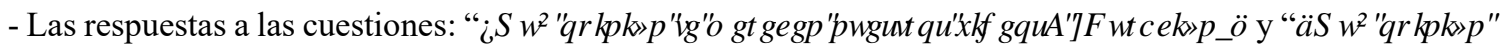

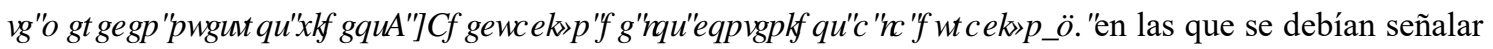
valoraciones, desde deficientes hasta excelentes, muestran una muy buena aceptación en relación a la duración y muy buena, aunque inferior, en relación a la adecuación (Fig. 6)..
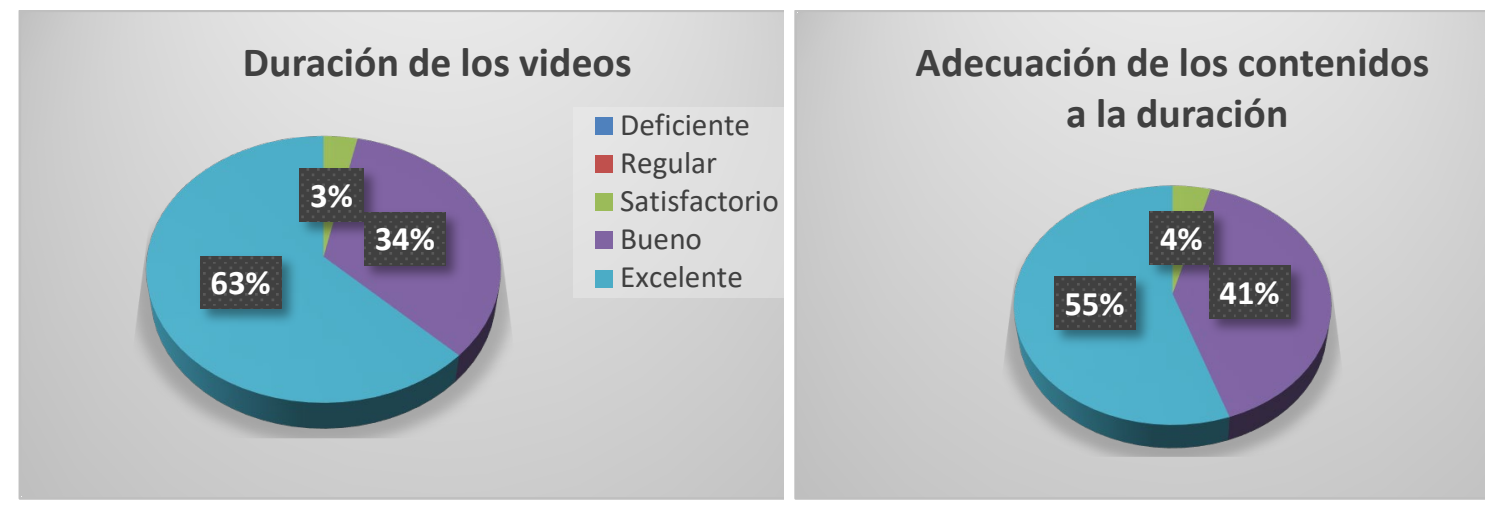

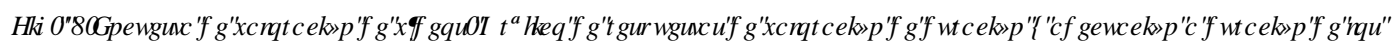
FRQMQICRV

- Las respuestas en cuanto a la percepción de calidad técnica de los videos, sonido e imagen, de las preguntas

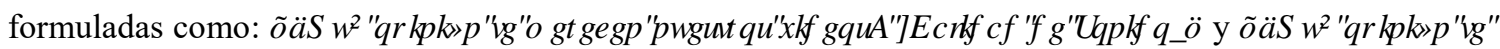

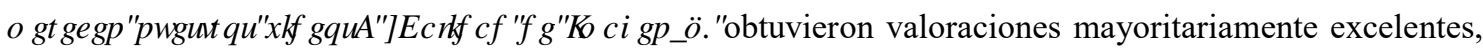
mejorables, según un $21 \%$ del alumnado, y no se estimaron regulares o deficientes (Fig. 6).. 

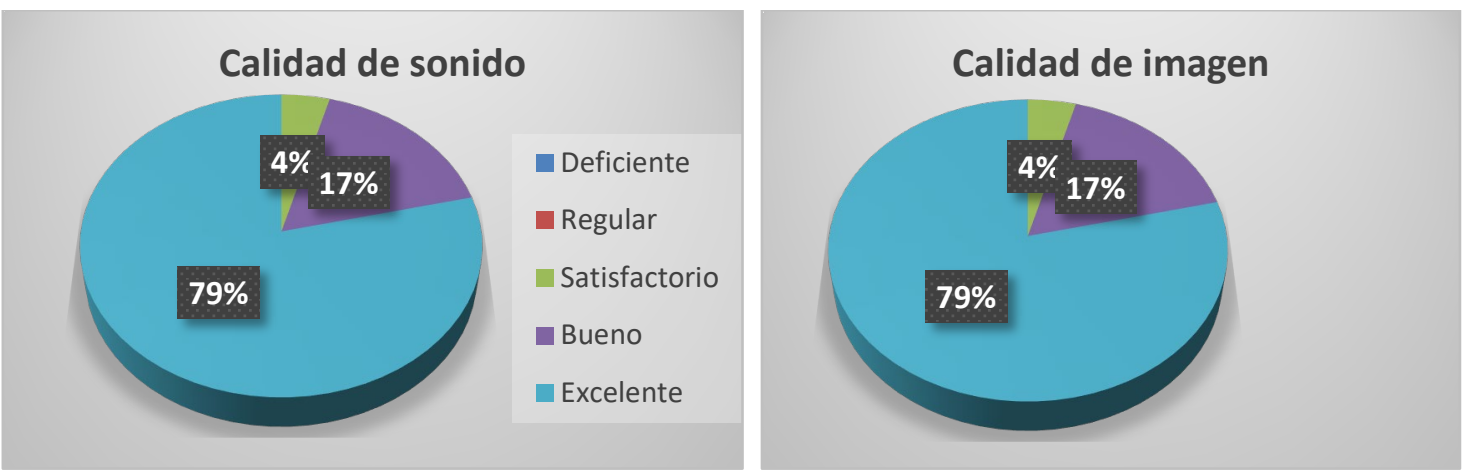

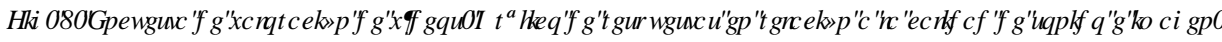

- Por último, las respuestas finales de esta segunda encuesta de valoración que, en sus preguntas hacían hincapié sobre la realidad de la utilidad de estos videos educativos (en la inicial se valoraba la expectativa).

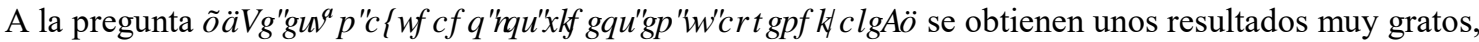
ya que ante las opciones de "son fundamentales, bastante, complementaria, poca o nada utilidad", mayoritariamente han contestado "son fundamentales" (66\%), seguida de "bastante" (34\%) y no se han obtenido valoraciones de "poca" o "nada" utilidad (Fig.7).

- Se ha contrastado esta utilidad verificada con la cuestión sobre la utilidad de una Guía de orientación del

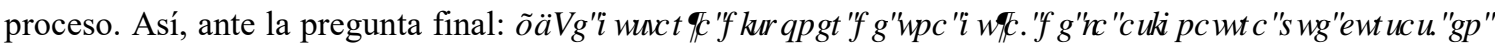

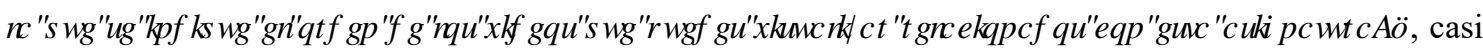
unánimemente (96\%) muestran su preferencia a que se incluya en el modelo de EA (Fig. 7).

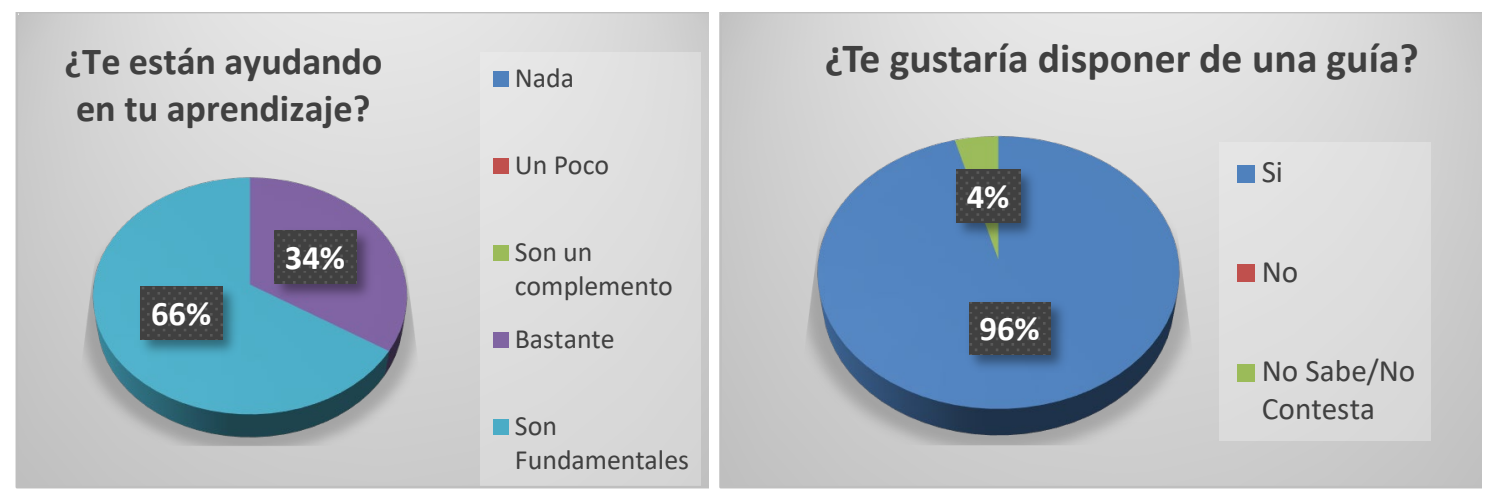

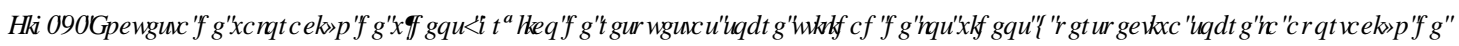
$X Q D * X+D D O P R G H R^{3}$ 9DXФ' प

- La valoración que recibe el canal por parte de la muestra es de una media superior a 9 (Fig. 4). 


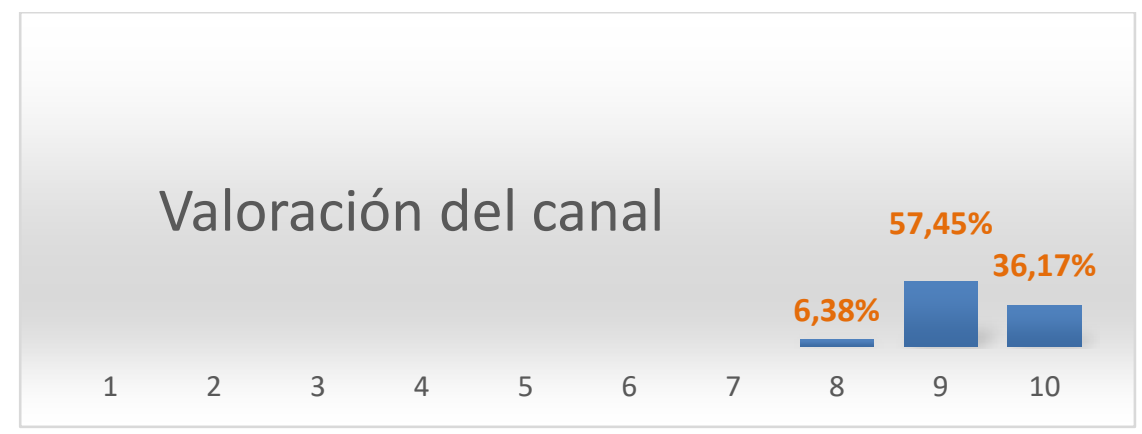

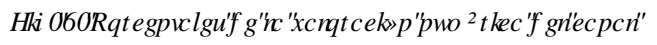

\section{Conclusiones}

Las respuestas de la encuesta inicial nos llevan a concluir que el alumnado mayoritariamente afirma la utilidad y necesidad de disponer de videos explicativos de los contenidos y problemas de la asignatura, junto con una guía de orientación relativa al recorrido educativo a seguir tanto en el canal, como en la asignatura. Esto, nos lleva a afirmar que las nuevas herramientas introducidas en nuestro modelo de EA, y, por tanto, las propias metodologías son aceptadas y consideradas de gran utilidad por el alumnado.

Con la información recabada, bien sea como respuesta a preguntas concretas o en el espacio abierto a comentarios, concluimos que el alumnado muestra su preferencia (90.2\%) por visionar los videos "a posteriori" de las explicaciones en clase que "a priori (76.1\%). También, que los problemas a resolver sean concretamente los propuestos en las relaciones de ejercicios.

Estas respuestas nos llevan a concluir que el alumnado prefiere utilizar su tiempo de autoaprendizaje en consolidar la enseñanza concreta que le damos en las clases magistrales y muestra menos interés en tener una visión más amplia de la materia con un autoaprendizaje previo o explorando otros aspectos.

A pesar de esa preferencia, no es desdeñable el porcentaje del $76 \%$ de participantes, en la encuesta, que aprueban el valor de prepararse "a priori" para la clase, con un visionado de videos recomendados. Se muestra de esta manera interés mayoritario por realizar un trabajo previo que ayuda a fortalecer el que posteriormente se realiza en clase.

La positiva valoración del canal de YouTube refuerza la utilidad de esta herramienta y consolida el modelo que estamos incluyendo en nuestra metodología docente.

Es destacable la concordancia y mejora entre los resultados obtenidos en la encuesta que se realizó en la etapa previa para detectar la expectativa de disponer de videos educativos para el aprendizaje, en la que el alumnado marcaba el $96.7 \%$ (Fig. 1), a, posteriormente, los resultados de la encuesta sobre la valoración de la utilidad de los videos (Fig.7), una vez materializados y visualizados, que supone una realidad del $100 \%$ dividido entre EDWDQWH(34\%) y VRQIXQGDP HQWDON (66\%).

La necesidad de una Guía orientativa con información complementaria es marcada como una expectativa útil, en la encuesta previa, por un 92.4\% (Fig.3). En la encuesta posterior a la puesta en marcha del canal, se demandaba por un $96 \%$ de los participantes. Estos datos confirman su pertinencia y utilidad.

Constatamos una demanda en el alumnado para que el profesorado complemente la docencia tradicional con herramientas TIC, como los videos didácticos. Esta demanda no está motivada sólo para que puedan 
disponer de material complementario, sino para ofrecer una alternativa clara y adecuada a sus estudios concretos.

Es una realidad que el alumnado, mayoritariamente, busca en internet este tipo de herramientas, Sin embargo, la información que encuentran es muy diversa, dispersa y no siempre adecuada y de calidad. Esta demanda, que todos conocemos, nos debe impulsar a ampliar este proyecto con contenidos que impartimos en nuestras asignaturas en la universidad, más allá de los de Cálculo numérico.

\section{Referencias}

CABERO, J. \& MARÍN, V. (2014). "Posibilidades educativas de las redes sociales y el trabajo en grupo. Percepciones

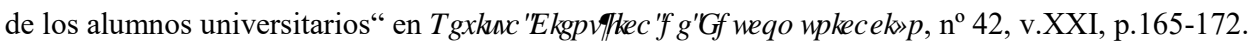

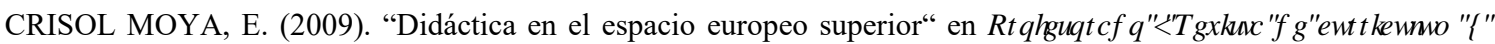
IRLPDFIYQ GHDSLRIHRWR, Vol.13, No 1, p. 387-394. Es reseña de : " RODRÍGUEZ FUENTES, A., CAURCEL CARA, M.J. y RAMOS GARCÍA, A.M.(Coords.) (2008). "Didáctica en el espacio europeo superior “.

CRISOL MOYA, E. \& ROMERO LÓPEZ, M.A. (2013). "Las guías de trabajo autónomo a través de Moodle. Opinión de los estudiantes. Una experiencia en la Universidad de Granada“" en 8 \&6\&. Vol.12, No. 23, enero-julio,2013. P. 159175

GÁMIZ, V., RODRÍGUEZ, M. J. y ROMERO, M. A. (2008). "Las herramientas didácticas en la universidad” en A. Rodríguez, M.J. Caurcel y A. M. Ramos (Coords), ' IG FUFDHQHD( ISDFIRI XURSHRIGH( GXFDFlyQ6XSHURUI* XtDVGGH WDEDNDXWYRP R. Madrid: EOS Universitaria, p. 71-92.

GARCÍA, A. y RODRÍGUEZ, A. (2008). "Las guías de trabajo autónomo en la universidad" en A. Rodríguez, M.J. Caurcel y A.M. Ramos (Coords.), ' IG FUFD HQ HD( ISDFIRI ( XURSHD GH ( GXFDFIY Q 6XSHURU * XtDV GH WDEDW DXWWRPR. Madrid: EOS Universitaria, p. 93-117.

GÓMEZ, M., ROSES, S. y FARIAS, P. (2012). "El uso académico de las redes sociales" en 5HLWD\&IHQWIFDGH ( GXFRP XQIFDFIy $\mathrm{n}^{\circ}$ 38, v. XIX, p. 131-138.

GUTIÉRREZ PORLÁN, I. (2011). "Aprendizaje con redes sociales : Tejidos educativos para los nuevos entornos“ en

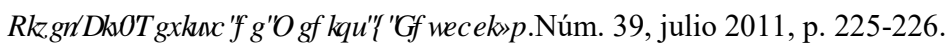

HUERTAS, A., MOLINA, M.F. y ROSALES, M.C. (2020). "La enseñanza universitaria mediante la combinación de técnicas tradicionales y estrategias basadas en las TIC“. En : [, QQRIDFIyQ ' RFHQUH H, QYHMWDFIy Q HQ \&IHQFDWD

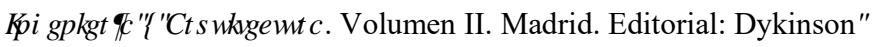

JIMÉNEZ CASTILLO, D. y MARÍN CARRILLO, G.M. (2012). “Asimilación de contenidos y aprendizaje mediante el uso de videotutoriales" en ( QMKDOJDD प7HDFKQJ, 30, 2-2012, p.63-79.

LÓPEZ-QUINTERO, J. L., PONTES-PEDRAJAS, A. y VARO-MARTÍNEZ, M. (2019ם"“Las TIC en la enseñanza científico-técnica hispanoamericana : Una revisión bibliográfica“. ' LILDO( GXFDURQ15HIIHZ-Number 35 June 2019$<$ http://greav.ub.edu/der/> [Consulta : 5 de febrero de 2021]

LOZANO DÍAZ, A., GONZÁLEZ MORENO, M.J. y CUENCA PIQUERAS, C. (2020). "Youtube comorecurso

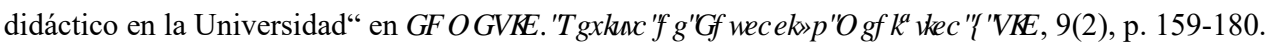

NAVARIDAS, NALDA, F., JIMÉNEZ TRENS, M. A. y FERNÁNDEZ ORTÍZ, R. (2016). "El aprendizaje de competencias en la Universidad : expectativas predictivas y niveles de confirmación de los estudiantes“ en Revista Española de Pedagogía. № 264, mayo-agosto 2016, p. 337-356.

RODRÍGUEZ FUENTES, A., CAURCEL CARA, M.J. y RAMOS GARCÍA, A.M.(Coords.) (2008). ' IG FUFDHQHD HSDFIRIHXLRSHRUSHURU Madrid : Editorial EOS.

ROMERO ARIZA, M. QUESADA ARMENTEROS, A. (2014). "Nuevas tecnologías y aprendizaje significativo de las ciencias" en ( QUKRDODDGHOVK\&IHPHDV, Núm.32.1, p.101-115.

ROMERO, M. A. \& CRISOL, E. (2009). "La guía de trabajo autónomo en la experiencia ECTS de la Titulación de

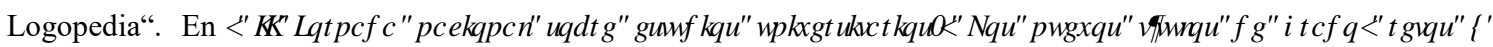
RSRUXQRGDAN. Castellón. Edita : Universitat Jaume I. Servei de Comunicació i Publicacions. Castellón. 125-126. 
ROMERO LÓPEZ, M. A. \& CRISOL MOYA, E. (2012). "Las guías de aprendizaje autónomo como herramienta didáctica de apoyo a la docencia" en ( VEXHDI\$ EIHUD, 15, p. 9-31.

SÁENZ BARRIO, O. (1994). "Métodos autodirectivos e individualizados"en Didáctica general : un enfoque curricular. Coord. Óscar Sáenz Barrio, p. 341-380.

VIDAL SALAZAR, M. D., FERRÓN VÍLCHEZ, V. y DE LA TORRE RUIZ, J.M. (2011). "La metodología del aprendizaje por indagación en la enseñanza universitaria : ejemplos concretos de aplicación“. Póster. En \&RQJUHR] , QHAQDFIRQDOGH, QQRIDFIy Q' RFHQH Cartagena. Cartagena : Universidad Politécnica de Cartagena, p.75

WEBB, M. E. (2011). "Affordances of ICT in science learning: implications for an integrated pedagogy" en ,QULQDURQDO-RXQQDORILFFHQFH( GXFDURQ 27:6, p.705-735. 